\title{
Secrecy-Oriented Optimization of Sparse Code Multiple Access for Simultaneous Wireless Information and Power Transfer in 6G Aerial Access Networks
}

\author{
Jingmin Zhang $\mathbb{D},{ }^{1,2}$ Xiaokui Yue, ${ }^{1}$ Xuan $\mathrm{Li}^{2}{ }^{2}$ Haofei Zhang, ${ }^{2}$ Tao $\mathrm{Ni}^{3}$ and Wensheng Lin $\mathbb{D}^{4}$ \\ ${ }^{1}$ School of Astronautics, Northwestern Polytechnical University, Xi'an 710072, China \\ ${ }^{2}$ No. 208 Research Institute of China Ordnance Industries, Beijing 102202, China \\ ${ }^{3}$ Shanghai Institute of Satellite Engineering, Shanghai 201109, China \\ ${ }^{4}$ School of Electronics and Information, Northwestern Polytechnical University, Xi'an 710072, China
}

Correspondence should be addressed to Wensheng Lin; linwest@nwpu.edu.cn

Received 5 March 2021; Revised 2 April 2021; Accepted 13 April 2021; Published 29 April 2021

Academic Editor: Haitao Xu

Copyright ( 2021 Jingmin Zhang et al. This is an open access article distributed under the Creative Commons Attribution License, which permits unrestricted use, distribution, and reproduction in any medium, provided the original work is properly cited.

\begin{abstract}
This article focuses on the simultaneous wireless information and power transfer (SWIPT) systems, which provide both the power supply and the communications for Internet-of-Things (IoT) devices in the sixth-generation (6G) network. Due to the extremely stringent requirements on reliability, speed, and security in the $6 \mathrm{G}$ network, aerial access networks (AANs) are deployed to extend the coverage of wireless communications and guarantee robustness. Moreover, sparse code multiple access (SCMA) is implemented on the SWIPT system to further promote the spectrum efficiency. To improve the speed and security of SWIPT systems in 6G AANs, we have developed an optimization algorithm of SCMA to maximize the secrecy sum rate (SSR). Specifically, a powersplitting (PS) strategy is applied by each user to coordinate its energy harvesting and information decoding. Hence, the SSR maximization problems in the SCMA system are formulated in terms of the PS and resource allocation, under the constraints on the minimum rates and minimum harvested energy of individual users. Then, a successive convex approximation method is introduced to transform the nonconvex problems to the convex ones, which are then solved by an iterative algorithm. In addition, we investigate the SSR performance of the SCMA system supported by our optimization methods, when the impacts from different perspectives are considered. Our studies and simulation results show that the SCMA system supported by our proposed optimization algorithms significantly outperforms the legacy system with uniform power allocation and fixed PS.
\end{abstract}

\section{Introduction}

It is universally acknowledged that with the wireless data traffic growing dramatically, the $6 \mathrm{G}$ networks have extremely stringent requirements on robustness, speed, and security [1, 2]. As a promising solution to guaranteeing reliability, AANs [3] can effectively extend the coverage of wireless communications and complement the terrestrial communication infrastructure. With the development of edge caching [4] and beam-steering [5] for multiple aerial platforms, AANs are enabled to provide services with even lower delay and more reliable connections. Supported by AAN, IoT will have higher flexibility for the deployment in complex environments. Furthermore, heterogeneous network technologies
$[6,7]$ enhance the performance of (IoT) systems, which allow more device connections than conventional systems. Definitely, an enormous number of IoT devices result in extraordinarily high cost for maintenance, such as recharging. Especially for those IoT devices deployed far away from the urban area, power supply is the key problem that determines the life of IoT devices. Fortunately, with the aid of AANs, the IoT devices can be recharged by wireless power transfer. Therefore, SWIPT by ANNs has great potential for providing power supply and internet connections to the IoT devices in the $6 \mathrm{G}$ era.

On the other hand, numerous IoT devices also demand massive connections, which should be supported by multiple access (MA) [8] without any doubt. According to references, 
MA may be operated in the form of orthogonal multiple access (OMA) or of nonorthogonal multiple access (NOMA). In comparison with OMA, NOMA enables multiple users to share one or a group of channels simultaneously. This property of NOMA has already been exploited in AAN [9]. According to the literature, there are typically two types of NOMA techniques, namely, power domain NOMA (PDNOMA) $[10,11]$ and code domain NOMA, such as sparse code multiple access (SCMA) [12]. Specifically, in the codebook-based SCMA, a set of subcarriers can be jointly assigned to a number of users via appropriate codebook assignment [12]. By adopting SCMA, we are able to further improve the spectrum efficiency. Therefore, this article focuses on SCMA for SWIPT in 6G AANs.

In the literature, SWIPT was initially proposed in [13] to achieve high energy efficiency, and it has attracted numerous related researches [14-21]. Specifically, in [18], a range of energy-harvesting schemes implemented with powersplitting (PS) and time-switching receivers was proposed. In these schemes, energy harvesting and information processing are operated in different domains at different time periods. The authors of [19] considered the NOMA systems with SWIFT and proposed a time-switching scheme motivating to maximize the sum rate and improve the fairness. A NOMA system with SWIPT was also investigated in [20], where a cooperative NOMA protocol was proposed to allow a user close to BS to assist a NOMA user far away from BS, in order for it to achieve energy harvesting. Furthermore, in [21], the authors investigated the trade-off between the achievable data rate and the obtainable harvested energy in the context of the SCMA systems functioning with SWIPT. Correspondingly, an optimization problem was designed to maximize the sum of weighted rate and energy and solved via joint $\mathrm{PA}$, codebook assignment, and PS.

Regarding SCMA, the previous works show that resource allocation (RA) can be invoked to significantly improve the performance of SCMA [22-24] systems. With this motivation, the authors in [22] addressed the sum rate of the SCMA systems with Gaussian inputs. Furthermore, a joint PA and codebook assignment algorithm was proposed, which allows achieving a suboptimal solution. Combining orthogonal frequency division multiple access (OFDMA) with SCMA, the authors of [23] proposed a low complexity subcarrierpairing scheme and an optimization strategy for the design of an uplink transmission [23]. Additionally, the authors in [24] proposed a novel PA scheme for the multiuser SCMA downlink systems with finite-alphabet inputs to improve the fairness of resource usage.

Besides the basic requirements on reliability and speed in the $6 \mathrm{G}$ networks, secure communications are also increasingly important in modern wireless communications. In recent years, the physical layer security (PLS) techniques, which provide security in wireless communications based on the characteristics of physical channels, have drawn wide research interest. In principle, PLS can achieve both secrecy and authentication simultaneously [25]. Hence, it is capable of providing some advantages over the conventional security techniques, e.g., cryptography [26]. In the context of the research on the PLS-enabled NOMA, e.g., the authors in
[27] investigated the PLS in a NOMA-assisted large-scale network, when assuming that users and eavesdroppers are randomly deployed. The studies show that via expanding either the zone of users or the size of the users' protected zone, the secrecy performance of the network can be improved. In [28], the principles of the NOMA-relied PLS considered in [27] were extended to the single-antenna and multiantenna large-scale networks. By contrast, in [29], the authors investigated the impact of a half-duplex relay (HDR) and a full-duplex relay (FDR) on the PLS of the NOMA systems, showing that the secrecy performance of the FDR-enabled NOMA systems is better than that of the HDR-assisted NOMA systems. The authors in [30] are motivated to maximize the secrecy sum rate (SSR) of NOMA systems under the constraint of the rate demanded by individual users. Furthermore, in [31], the secrecy rate maximization in the multiple-input-single-output systems experiencing a multiple-antenna eavesdropper was considered, where multiple randomly located jammers are introduced to enhance the secrecy performance. Additionally, the SSR performance of the NOMA systems with SWIPT was considered in [32].

Nevertheless, to the best of our knowledge, PLS has not been investigated in conjunction with the SCMA systems enabling SWIPT, while mostly related references considered the codebook or detector design of SCMA systems. In the literature, a range of researches on PD-NOMA, such as $[19,27-$ 30], considered PLS or SWIPT. Furthermore, in [33], the sum rate performance between PD-NOMA and SCMA systems was compared, but without invoking SWIPT. Therefore, in this article, we are motivated to investigate the PLS design in the PD-NOMA and SCMA systems providing SWIPT services. As a result, we derive the optimal RA and PS under the constraints on the minimum rates and minimum harvested power of individual users, in order to maximize the overall SSR of the SCMA systems.

To be more specific, users in the SCMA systems are assumed to have no energy stored, and hence, they have to harvest energy, such as for decoding of received information. In this case, the SSR maximization problem needs to be formulated under the constraints on the minimum rates and minimum harvested energy of individual users. Correspondingly, the optimization problem for the SCMA system includes the PS, codebook assignment, and PA. Hence, jointly solving this problem is highly involved. In order to make the problem approachable with desirable solutions, the successive convex approximation (SCA) method is introduced, based on which, we propose an iterative algorithm for deriving the solutions to the problem. As the algorithm name implies, the subproblems for the PS, codebook assignment, and PA are solved separately but in an iterative way. Specifically, the algorithm first derives the closed-form solution for the PS, under the assumption of a given PA and codebook assignment. Then, based on the obtained PS, the codebook assignment subproblem is addressed via transforming it to a geometric programming (GP) problem, which is obtained with the aid of the arithmetic geometric mean approximation (AGMA) [34]. Finally, for the given PS and codebook assignment as obtained above, we can transform the PA subproblem to a convex problem upon following the principles of 
the SCA for low complexity (SCALE) method [35]. Since the resulted problem is convex and satisfies Slater's condition [36], it can be solved by the subgradient method [21], after transforming it to a dual problem.

The main contributions of this article are summarized as follows:

(i) We have established a framework for the optimization of SWIPT with SCMA in 6G AANs, where the PLS is investigated in-depth. This framework provides the methodology for the design of IoT systems supported by $6 \mathrm{G}$ AANs

(ii) An iterative algorithm is proposed for solving the SSR maximization problem in the SCMA systems providing SWIPT. With the iterative algorithm, the PS, codebook assignment, and the PA subproblems are separately solved but iteratively, so that nearoptimum SSR performance can be achieved with significantly reduced complexity

(iii) Finally, the SSR performance of the SCMA systems is investigated from different perspectives associated with the requirements of the minimum rates and the minimum harvested energy of individual users. The results verify that our proposed optimization framework distinctly outperforms the legacy system

The rest of this article is organized as follows. Section 2 describes the system model to be studied and then formulates the optimization problem for SSR maximization. In Section 3 , the optimal PS, codebook assignment, and PA in the SCMA systems providing SWIPT are addressed. Simulation results are demonstrated in Section 4 to show the impact of different parameters on the SSR performance of the system. Finally, observations from this research are summarized in Section 5 .

\section{System Model and Problem Formulation}

2.1. System Model. Figure 1 illustrates the system model of secrecy SWIPT with SCMA in 6G AANs. We consider a single-cell downlink of SWIPT, which is constituted by one base station (BS) supporting $M$ legitimate users and eavesdropped by one passive eavesdropper. Assume that the BS can measure and know the CSI of all legitimate users, while the CSI of the eavesdropper is unknown to the BS, as it is a passive eavesdropper. The channel gains of the legitimate users and of the eavesdropper are denoted as $h_{m}$ $(m=1,2, \cdots, M)$ and $h_{e}$, respectively.

Moreover, the users are empowered with the energyharvesting function for harvesting energy from wireless channels based on a PS strategy having the architecture depicted in Figure 2. According to this PS scheme, signals sent by BS to users are split into two streams, one of which is for energy harvesting, and the other is for information delivery. The PS ratios of the $M$ users are expressed as $\rho=\{$ $\left.\rho_{1}, \rho_{2} \cdots, \rho_{M}\right\}$. We assume that at a user, the harvested energy is stored in its chargeable batteries. For the eavesdropper, we assume that it does not harvest energy from wireless chan-

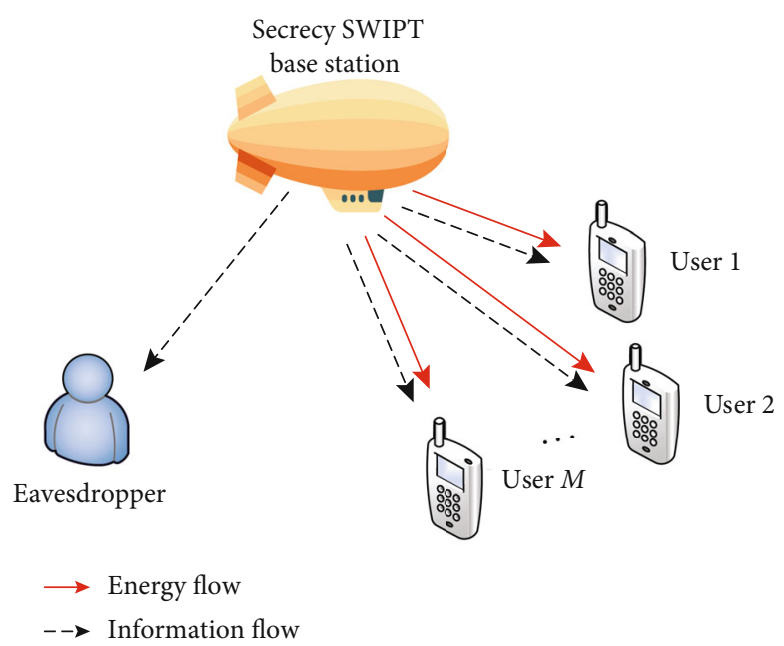

Figure 1: Secrecy SWIPT with SCMA in 6G AANs.

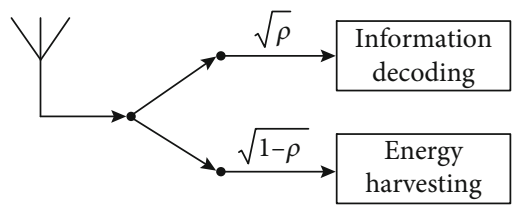

Figure 2: Power-splitting architecture of mobile users.

nels. Furthermore, we assume that every node in the system, including BS, users, and eavesdropper, is equipped with a single omnidirectional antenna. Based on these assumptions, the problem of SSR maximization in the SCMA system is formulated below.

2.2. Problem Formulation. In the principles of SCMA [37], we assume that BS sends information to the $M$ users using in total $C$ codebooks and $N$ subcarriers. Assume that the codebook size for each user is $B$, and the length of codewords is $N$. Then, BS is able to deliver $\log _{2} B$ bits per symbol to a user. Specifically, to transmit a symbol to the $m$-th user, a bit sequence $\mathbf{b}_{m}$ of length $\log _{2} B$ is mapped to a SCMA codeword expressed as $\mathbf{x}_{m, c}=\left[x_{m, c}^{1}, x_{m, c}^{2}, \cdots, x_{m, c}^{N}\right]^{T}$. Here, the index $c$ denotes that the codeword is chosen from the $c$-th codebook predefined. Note that $x_{m, c}^{n}$ is a complex symbol, which is transmitted on the $n$-th subcarrier. Furthermore, let us denote the sparsity of the $N$-length codewords as $L$, i.e., the codewords are $N$-length vectors with $L(L<N)$ nonzero entries. Since the $L$ nonzero entries of a codeword correspond to $L$-specific subcarriers activated, we refer to such a codeword as the $L$-specific subcarriers for convenience.

Let us denote $\mathbf{p}=\left\{q_{c}^{n}\right\}$ as the mapping relationship between a codebook and the $N$ subcarriers, where $q_{c}^{n}=1$ if codebook $c$ occupies subcarrier $n$; otherwise, $q_{c}^{n}=0$. Let us denote $\mathbf{S}=\left\{s_{m, c}\right\}$ as the codebook assignment indicator, where $s_{m, c}=1$ when codebook $c$ is assigned to user $m$, and $s_{m, c}=0$, otherwise. Furthermore, the power allocation is expressed as $\mathbf{P}=\left\{p_{m, c}\right\}$, where $p_{m, c}$ denotes the transmit power, if user $m$ is assigned codebook $c$. Based on these 
definitions, the total transmit power of BS is $P_{s}=\sum_{m=1}^{M} \sum_{c=1}^{C}$ $s_{m, c} p_{m, c}$

When user $m$ is assigned the codebook $c$, it has the total transmit power $p_{m, c}$, which is then assigned to the $L$-active subcarriers. Let the PA coefficients be expressed as $\left\{\alpha_{c}^{n}\right\}$, where $0<\alpha_{c}^{n}<1$ when $q_{c}^{n}=1$ (subcarrier $n$ active), and $\alpha_{c}^{n}$ $=0$ if $q_{c}^{n}=0$ (subcarrier $n$ inactive). In principle, more than one codebook may be assigned to one user, but a given codebook will not be simultaneously assigned to more than one user. However, for the problem to be manageable, in this article, we assume the one-to-one assignment, meaning that one user is only assigned one codebook.

Based on the above definitions, at the $m$-th user, the received signal on the $n$-th subcarrier can be expressed as

$$
y_{m, c}^{n}=h_{m}^{n} \sqrt{s_{m, c} p_{m, c} \alpha_{c}^{n}} x_{m, c}^{n}+w_{m}^{n},
$$

where $w_{m}^{n} \sim C N\left(0, \sigma^{2}\right)$ is the Gaussian noise introduced by the radio frequency to baseband conversion (RFBC) operation [38]. When considering the simultaneous transmission of information and energy, the component signal used to decode information can be expressed as

$$
y_{\mathrm{m}, c}^{n, I}=\sqrt{\rho_{m}}\left(h_{m}^{n} \sqrt{s_{m, c} p_{m, c} \alpha_{c}^{n}} x_{m, c}^{n}\right)+w_{m}^{n}
$$

while the component signal used for energy harvesting can be expressed as

$$
y_{m, c}^{n, H}=\sqrt{1-\rho_{m}}\left(h_{m}^{n} \sqrt{s_{m, c} p_{m, c} \alpha_{c}^{n}} x_{m, c}^{n}\right)+w_{m}^{n} .
$$

According to the principles of SCMA, the message passing algorithm (MPA) [39] can be employed by users to achieve near-optimum performance. By contrast, for energy harvesting, when both codeword assignment and PA are taken into account, the energy harvested by the $m$-th user can be found to be

$E_{m}=\eta\left(1-\rho_{m}\right) \sum_{m}^{\prime=1} M \sum_{c=1}^{C} \sum_{n=1}^{N} \alpha_{c}^{n} p_{m}{ }^{l, c} s_{m}{ }^{l, c}\left|h_{m}^{n}\right|^{2}, \quad m=1,2, \cdots, M$,

where $\eta(0<\eta \leq 1)$ stands for the efficiency of energy harvesting.

The signal received by the eavesdropper for wiretapping the $m$-th user can be expressed as

$$
y_{e, c}^{n, m}=h_{e}^{n} \sqrt{s_{m, c} p_{m, c} \alpha_{c}^{n}} x_{m, c}^{n}+w_{e}^{n}, \quad n=1,2, \cdots, N .
$$

We assume that the eavesdropper is capable of obtaining all the codebooks, so that it is capable of intercepting each user's message.

From (2) and (5), the signal-to-noise ratio (SNR) of subcarrier $n$ for user $m$ and the SNR of subcarrier $n$ for the eavesdropper can be expressed as

$$
\gamma_{m, c}^{n}=\rho_{m} \alpha_{c}^{n} s_{m, c} p_{m, c}\left|h_{m}^{n}\right|^{2} \sigma^{-2}
$$

$$
\gamma_{e, c}^{n, m}=\alpha_{c}^{n} s_{m, c} p_{m, c}\left|h_{e}^{n}\right|^{2} \sigma^{-2},
$$

respectively. Therefore, the sum rate attainable by the $m$-th user and that achievable by the eavesdropper for wiretapping the $m$-th user can be expressed as

$$
\begin{gathered}
R_{m}=\sum_{c=1}^{C} \sum_{n=1}^{N} \log _{2}\left(1+\gamma_{m, c}^{n}\right), \quad m=1,2, \cdots, M, \\
R_{e}^{m}=\sum_{c=1}^{C} \sum_{n=1}^{N} \log _{2}\left(1+\gamma_{e, c}^{n, m}\right), \quad m=1,2, \cdots, M,
\end{gathered}
$$

respectively. Consequently, we can obtain the achievable secrecy rate of user $m$ and the SSR of the SCMA downlink system as

$$
\begin{gathered}
R_{m}^{s}=\sum_{c=1}^{C} \sum_{n=1}^{N}\left[\log _{2}\left(1+\gamma_{m, c}^{n}\right)-\log _{2}\left(1+\gamma_{e, c}^{n, m}\right)\right]^{+}, \\
R_{s}=\sum_{m=1}^{M} R_{m}^{s},
\end{gathered}
$$

respectively.

From (6), (7), (8), and (9), we can readily know that when $\gamma_{m, c}^{n} \leq \gamma_{e, c}^{n, m}$, the secrecy rate transmitting on subcarrier $n$ of user $m$ is zero. Furthermore, we can derive that $\gamma_{m, c}^{n} \leq \gamma_{e, c}^{n, m}$, provided that $\left|h_{m}^{n}\right|^{2} \leq\left|h_{e}^{n}\right|^{2} / \rho_{m}$. Therefore, without loss of generality, we can arrange the channel gains in ascending order as $0<\left|h_{1}^{n}\right|^{2} \leq\left|h_{2}^{n}\right|^{2} \leq \cdots \leq\left|h_{M_{e}^{n}}^{n}\right|^{2} \leq\left|h_{e}^{n}\right|^{2} / \rho_{m}<\left|h_{M_{e}^{n}+1}^{n}\right|^{2} \leq$ $\cdots \leq\left|h_{M}^{n}\right|^{2}$ for the $n$-th subcarrier. Then, $R_{s}$ of (11) can be rewritten as

$$
R_{s}=\sum_{c=1}^{C} \sum_{n=1}^{N} \sum_{m=M_{e}^{n}+1}^{M}\left[\frac{\log _{2}\left(1+\gamma_{m, c}^{n}\right)}{\log _{2}\left(1+\gamma_{e, c}^{n, m}\right)}\right]
$$

For SWIPT, we assume that each user also has the requirements for the minimum harvested energy $E_{m}^{\text {req }}$ and the minimum required data rate $R_{m}^{\text {req }}$. Correspondingly, the SSR maximization problem for the SCMA system can be described as

$$
\begin{gathered}
\max _{\rho, \mathbf{S}, \mathbf{P}} R_{s}, \\
\text { s.t. } R_{m} \geq R_{m}^{\mathrm{req}}, \quad \forall m, \\
E_{m} \geq E_{m}^{\mathrm{req}}, \quad \forall m, \\
\sum_{m=1}^{M} \sum_{c=1}^{C} s_{m, c} p_{m, c}=P_{s}, \\
\sum_{m=1}^{M} s_{m, c}=1, \sum_{c=1}^{C} s_{m, c}=1, s_{m, c} \in\{0,1\}, \quad \forall m, c .
\end{gathered}
$$

Note that the constraints in (13e) infer that one user is only assigned one codebook. 


\section{SSR Optimization of SCMA Systems}

According to (13a), (13b), (13c), (13d), and (13e), we can know that the SSR optimization of SCMA systems is a mixed-integer nonconvex problem. In order to solve this problem, in this section, an iterative algorithm is proposed to optimize the PS, codebook assignment, and PA. To summarize, at each iteration, we first derive the PS ratios according to the monotonicity of the objective function in (13a) under a given RA strategy for the codebook and PA. Then, the codebook is assigned by applying the AGMA method [34]. Finally, the SCALE algorithm [35] is introduced for the PA.

3.1. Power-Splitting Ratio Selection. In order to obtain the optimum PS ratios, let us first introduce the following theorem.

Theorem 1. Given a RA strategy of $\{P, S\}$, when the maximum sum rate $R_{s}$ expressed as (13a) is attained, the constraints in (13c) should achieve the equality, yielding

$$
\rho_{m}=1-\frac{E_{m}^{r e q}}{\eta \sum_{m^{\prime}=1}^{M} \sum_{c=1}^{C} \sum_{n=1}^{N} \alpha_{c}^{n} p_{m}{ }^{\prime}{ }^{\prime} s_{m}{ }^{\prime}, c\left|h_{m}^{n}\right|^{2}}, \quad m=1, \cdots, M .
$$

Proof. First, from (12), we can prove that the maximum $R_{s}$ is achieved, if $\rho_{m}$ for $m=1,2, \cdots, M$ are set to their maximum possible values. This can be seen from (6) and (7) that $\gamma_{m, c}^{n}$ increases with the increase of $\rho_{m}$, and $\gamma_{e, c}^{n}$ is independent of $\rho_{m}$. Second, substituting (4) into (13c) yields the constraint on $\rho_{m}$ as

$$
\rho_{m} \leq 1-\frac{E_{m}^{r e q}}{\eta \sum_{m}^{M} \sum_{c=1}^{C} \sum_{c=1}^{C} \sum_{n=1}^{N} \alpha_{c}^{n} p_{m}{ }^{\prime}, c_{m}{ }^{\prime}, c\left|h_{m}^{n}\right|^{2}} .
$$

Therefore, in order to maximize (13a), we have the PS ratios given by (14). This completes the proof.

Having obtained the optimum PS ratios under the given RA strategy $\{P, S\}$, let us now consider the codebook assignment.

3.2. Codebook Assignment. The optimization problem of (13a), (13b), (13c), (13d), and (13e) becomes a codebook assignment, when $\rho$ and $P$ are fixed. In order to solve this problem, we relax $s_{m, c}$ seen in (13a), (13b), (13c), (13d), and (13e) to the real variables taking values between zero and one, i.e., $s_{m, c} \in[0,1]$, which can be interpreted as the fraction of time that codebook $c$ is assigned to user $m$. Moreover, without loss of any generality, we order the user's channel gains of $N$ subcarriers and eavesdropper's channel gains of $N$ subcarriers, as well as the mapping matrix between codebooks and subcarriers according to the value of $M_{e}^{n}$ as $\tilde{H}=$ $\left\{\tilde{h}_{m}^{n}\right\}, \tilde{H}_{e}=\left\{\tilde{h}_{e}^{n}\right\}$, and $\tilde{q}=\left\{\tilde{q}_{c}^{n}\right\}$, so that we have $M_{e}^{1} \leq M_{e}^{2} \leq$ $\cdots \leq M_{e}^{N}$. Then, from (6), (7), and (12), the optimization problem of (13a), (13b), (13c), (13d), and (13e) can be simplified to the following nonconvex problem:

$$
\begin{gathered}
\max _{S} \sum_{n=1}^{N} \sum_{c=1}^{C} \sum_{m=M_{e}^{n}+1}^{N} \log _{2}\left(\frac{\rho_{m} \tilde{\alpha}_{c}^{n} s_{m, c} p_{m, c}\left|\tilde{h}_{m}^{n}\right|^{2}+\sigma^{2}}{\tilde{\alpha}_{c}^{n} s_{m, c} p_{m, c}\left|\tilde{h}_{e}^{n}\right|^{2}+\sigma^{2}}\right), \\
\text { s.t. } \quad 0 \leq s_{m, c} \leq 1, \quad \forall m, c, \\
\text { s.t. } \quad(13 \mathrm{~b}),(13 \mathrm{c}),(13 \mathrm{~d}) \text {, and }(13 \mathrm{e}) .
\end{gathered}
$$

Below, we introduce the AGMA algorithm [34] to solve the codebook assignment problem. For this purpose, we rewrite the objective function in (16a) as

$$
\min _{S} \prod_{n=1}^{N} \prod_{c=1}^{C} \prod_{m=M_{e}^{n}+1}^{M} \frac{\tilde{\alpha}_{c}^{n} s_{m, c} p_{m, c}\left|\tilde{h}_{e}^{n}\right|^{2}+\sigma^{2}}{\rho_{m} \tilde{\alpha}_{c}^{n} s_{m, c} p_{m, c}\left|\tilde{h}_{m}^{n}\right|^{2}+\sigma^{2}} .
$$

Furthermore, we will make use of the following lemma from [34].

Lemma 2. If $g(x)=\sum_{i} u_{i}(x)$ is a posynomial (a posynomial is a function in the form of $f\left(x_{1}, \cdots, x_{n}\right)=\sum_{l=1}^{L} c_{l} x_{1}^{a_{1 l}} \cdots x_{n}^{a_{n l}}$, where $\left\{x_{i}\right\}$ and $\left\{c_{l}\right\}$ are all positive real numbers, while the exponents $\left\{a_{i l}\right\}$ are real numbers), then

$$
g(x) \geq \tilde{g}(x)=\prod_{i}\left(\frac{u_{i}(x)}{\kappa_{i}}\right)^{\kappa_{i}}
$$

where $\kappa_{i}=u_{i}\left(x_{0}\right) / g\left(x_{0}\right), \forall i$, and $x_{0}$ can be any fixed positive value satisfying $g\left(x_{0}\right)=\tilde{g}\left(x_{0}\right)$.

According to Lemma 2, let us define $X=\rho_{m} \tilde{\alpha}_{c}^{n} s_{m, c} p_{m, c}$ $\left|\tilde{h}_{m}^{n}\right|^{2}+\sigma^{2}$. Then, we can have the relationship of

$$
X \geq \tilde{X}=\left(\frac{\rho_{m} \tilde{\alpha}_{c}^{n} s_{m, c} p_{m, c}\left|\tilde{h}_{m}^{n}\right|^{2}}{\tau_{m, c}^{n}}\right)^{\tau_{m, c}^{n}}\left(\frac{\sigma^{2}}{v_{m, c}^{n}}\right)^{v_{m, c}^{n}}
$$

where $\tau_{m, c}^{n}=\rho_{m} \tilde{\alpha}_{c}^{n} s_{m, c}^{0} p_{m, c}\left|\tilde{h}_{m}^{n}\right|^{2} / X_{0}$ and $v_{m, c}^{n}=\sigma^{2} / X_{0}$. With the aid of this relationship, the objective function seen in (17) can be expressed as

$$
\prod_{n=1}^{N} \prod_{c=1}^{C} \prod_{m=M_{e}^{n}+1}^{M} \frac{\tilde{\alpha}_{c}^{n} s_{m, c} p_{m, c}\left|\tilde{h}_{e}^{n}\right|^{2}+\sigma^{2}}{\tilde{X}} .
$$

Similarly, we can exploit Lemma 2 to transform the constraint of (13b) to the convex form. Based on these transformations, consequently, the codebook assignment problem of 
(16a) and (16b) can be reformulated as

$$
\begin{gathered}
\min _{S} \prod_{n=1}^{N} \prod_{c=1}^{C} \prod_{m=M_{e}^{n}+1}^{M} \frac{\tilde{\alpha}_{c}^{n} s_{m, c} p_{m, c}\left|\tilde{h}_{e}^{n}\right|^{2}+\sigma^{2}}{\tilde{X}}, \\
\text { s.t. } \quad \prod_{n=1}^{N} \prod_{c=1}^{C} \frac{\tilde{X}}{\sigma^{2}} \geq 2^{R_{m}^{\text {req }}}, \quad \forall m, \\
\text { s.t. } \quad 0 \leq s_{m, c} \leq 1, \quad \forall m, c, \\
\text { s.t. } \quad(13 \mathrm{c}),(13 \mathrm{~d}), \text { and }(13 \mathrm{e}),
\end{gathered}
$$

which is a geometric programming (GP) problem [34] that can be efficiently solved by the available optimization toolboxes, e.g., the CVX toolbox [36].

3.3. Power Allocation. After the PS of $\rho$ and codebook assignment of $S$ are fixed, we now optimize the PA of SCMA systems. In this case, with the aid of (6), (7), and (12), the optimization problem for PA can be simplified from (13a), (13b), (13c), (13d), and (13e) as

$\max _{P} \sum_{n=1}^{N} \sum_{c=1}^{C} \sum_{m=M_{e}^{n}+1}^{M} \log _{2}\left(1+\frac{\tilde{\alpha}_{c}^{n} s_{m, c} p_{m, c}\left(\rho_{m}\left|\tilde{h}_{m}^{n}\right|^{2}-\left|\tilde{h}_{e}^{n}\right|^{2}\right)}{\tilde{\alpha}_{c}^{n} s_{m, c} p_{m, c}\left|\tilde{h}_{e}^{n}\right|^{2}+\sigma^{2}}\right)$

$$
\text { s.t. } \quad(13 b),(13 c) \text {, and }(13 d) \text {, }
$$

which is also nonconvex. However, it can be closely approximated using a convex problem obtained by the SCALE algorithm [35], which is detailed below.

First, let us consider a tight lower bound [35] of $u \log ($ $z)+v \leq \log (1+z)$, where $u=z_{0} /\left(z_{0}+1\right)$ and $v=\log \left(z_{0}+1\right.$ )$-\left(z_{0} /\left(z_{0}+1\right)\right) \log \left(z_{0}\right)$. Then, the optimization problem of (22a) and (22b) can be reformulated as

$\max _{P} \sum_{n=1}^{N} \sum_{c=1}^{C} \sum_{m=M_{e}^{n}+1}^{M} u_{m, c}^{n} \log _{2}\left(\frac{\tilde{\alpha}_{c}^{n} s_{m, c} p_{m, c}\left(\rho_{m}\left|\tilde{h}_{m}^{n}\right|^{2}-\left|\tilde{h}_{e}^{n}\right|^{2}\right)}{\tilde{\alpha}_{c}^{n} s_{m, c} p_{m, c}\left|\tilde{h}_{e}^{n}\right|^{2}+\sigma^{2}}\right)+v_{m, c}^{n}$,

$$
\text { s.t. }(13 b),(13 c) \text {, and }(13 d) \text {. }
$$

However, the optimization problem of (23a) is still nonconvex. In order to convert it to a convex one, we introduce the following lemma.

Lemma 3 (see [35]). The problem of (23a) can be converted to a convex problem by the transformation of $p_{m, c}=2^{\tilde{p}_{m, c}}$.
Correspondingly, the optimization problem of (23a) can now be stated as a convex problem as

$$
\begin{aligned}
\max _{P} & \sum_{n=1}^{N} \sum_{c=1}^{C} \sum_{m=M_{e}^{n}+1}^{M} u_{m, c}^{n}\left[\tilde{p}_{m, c}+\log _{2} \tilde{\alpha}_{c}^{n} s_{m, c}\left(\rho_{m}\left|\tilde{h}_{m}^{n}\right|^{2}-\left|\tilde{h}_{e}^{n}\right|^{2}\right)\right. \\
& \left.-\log _{2}\left(\tilde{\alpha}_{c}^{n} s_{m, c} 2^{\tilde{p}_{m, c}}\left|\tilde{h}_{e}^{n}\right|^{2}+\sigma^{2}\right)\right]+v_{m, c}^{n},
\end{aligned}
$$

$$
\text { s.t. } \sum_{c=1}^{C} \sum_{n=1}^{N} \log _{2}\left(1+\frac{\rho_{m} \tilde{\alpha}_{c}^{n} s_{m, c} \tilde{p}^{\tilde{p}_{m, c}}\left|\tilde{h}_{m}^{n}\right|^{2}}{\sigma^{2}}\right) \geq R_{m}^{r e q}, \quad \forall m,
$$

$$
\eta\left(1-\rho_{m}\right) \sum_{m}^{\prime=1} M \sum_{c=1}^{C} \sum_{n=1}^{N} \tilde{\alpha}_{c}^{n} 2^{\tilde{p}_{m^{\prime}, c}}{ }^{{ }_{m}}{ }^{\prime, c}\left|\tilde{h}_{m}^{n}\right|^{2} \geq E_{m}^{r e q}, \quad \forall m,
$$

$$
\sum_{m=1}^{M} \sum_{c=1}^{C} s_{m, c} 2^{\tilde{p}_{m, c}}=P_{s}
$$

Since the problem of (24a), (23b), (24c), and (24d) is convex, it satisfies Slater's condition [21]. Hence, the duality gap is zero, and the optimal PA can be obtained by solving the dual problem, with the Lagrangian function of

$$
\begin{aligned}
& L(P, \mu, \varsigma, \lambda)=\sum_{n=1}^{N} \sum_{c=1}^{C} \sum_{m=M_{e}^{n}+1}^{M}\left[u_{m, n}^{n} \tilde{c}_{m, c}+u_{m, c}^{n} \log _{2} \tilde{\alpha}_{c}^{n} s_{m, c}\right. \\
& \cdot\left(\rho_{m}\left|\tilde{h}_{m}^{n}\right|^{2}-\left|\tilde{h}_{e}^{n}\right|^{2}\right)-u_{m, c}^{n} \log _{2}\left(\tilde{\alpha}_{c}^{n} s_{m, c} 2^{\tilde{p}_{m, c}}\left|\tilde{h}_{e}^{n}\right|^{2}+\sigma^{2}\right) \\
& \left.+v_{m, c}^{n}\right]+\sum_{m=1}^{M} \mu_{m}\left[R_{m}^{r e q}-\sum_{c=1}^{C} \sum_{n=1}^{N} \log _{2}\right. \\
& \left.\cdot\left(1+\frac{\rho_{m} \tilde{\alpha}_{c}^{n} s_{m, c} 2^{\tilde{p}_{m, c}}\left|\tilde{h}_{m}^{n}\right|^{2}}{\sigma^{2}}\right)\right]+\sum_{m=1}^{M} \varsigma_{m} \\
& \cdot\left[E_{m}^{\mathrm{req}}-\eta\left(1-\rho_{m}\right) \sum_{m}^{\prime=1} M \sum_{c=1}^{C} \sum_{n=1}^{N} \tilde{\alpha}_{c}^{n} 2^{\tilde{p}_{m^{\prime}, c}} s_{m}{ }^{\prime, c}\left|\tilde{h}_{m}^{n}\right|^{2}\right] \lambda \\
& \left(\sum_{m=1}^{M} \sum_{c=1}^{C} s_{m, c} 2^{\tilde{p}_{m, c}}-P_{s}\right)
\end{aligned}
$$

where $\tilde{\mathbf{P}}=\left\{\tilde{p}_{m, c}\right\}, \mu=\left(\mu_{1}, \mu_{2}, \cdots, \mu_{M}\right), \varsigma=\left(\varsigma_{1}, \varsigma_{2}, \cdots, \varsigma_{M}\right)$, and $\lambda$ are the Lagrange multipliers for the constraints shown in (23b), (24c), and (24d), respectively. The dual function can be expressed as

$$
g(\mu, \varsigma, \lambda)=\max _{\tilde{\mathbf{P}}} L(\tilde{\mathbf{P}}, \mu, \varsigma, \lambda)
$$

and correspondingly, the dual problem can be stated as

$$
\min _{\mu, \varsigma, \lambda} g(\mu, \varsigma, \lambda)
$$


In order to solve the problem, we take the first-order partial derivative of the Lagrangian function of (25) with respect to $\tilde{p}_{m, c}$ for all $m$ and $c$, yielding

$$
\begin{aligned}
\frac{\partial L(\tilde{\mathbf{P}}, \mu, \varsigma, \lambda)}{\partial \tilde{p}_{m, c}}= & \sum_{n=1}^{N-N_{e}^{m}} u_{m, c}^{n} \frac{\sigma^{2}}{\tilde{\alpha}_{c}^{n} s_{m, c} p_{m, c}\left|\tilde{h}_{e}^{n}\right|^{2}+\sigma^{2}} \\
& -\sum_{n=1}^{N} \mu_{m} \frac{\rho_{m} \tilde{\alpha}_{c}^{n} s_{m, c} p_{m, c}\left|\tilde{h}_{m}^{n}\right|^{2}}{\rho_{m} \tilde{\alpha}_{c}^{n} s_{m, c} p_{m, c}\left|\tilde{h}_{m}^{n}\right|^{2}+\sigma^{2}} \\
& -\eta \ln 2 \sum_{m^{\prime}=1}^{M} \sum_{n=1}^{N} \varsigma_{m^{\prime}}\left(1-\rho_{m^{\prime}}\right) s_{m, c} p_{m, c} \tilde{\alpha}_{c}^{n}\left|\tilde{h}_{m^{\prime}}^{n}\right|^{2} \\
& +\lambda s_{m, c} p_{m, c} \ln 2,
\end{aligned}
$$

where $N_{e}^{m}$ is the number of $M_{e}^{n^{\prime}}$ s for $n=1,2, \cdots, N$ that is larger than or equal to $m$. According to the KKT conditions, when at the optimum points, (28) should be equal to 0 . However, in the general, solving the above equation is highly involved. Therefore, we impose the approximation on (28) by assuming that $\sigma^{2} \rightarrow 0$, meaning that the SNR is sufficiently high. In this case, from (28) and the necessary conditions for optimality, we can have

$$
a p_{m, c}^{2}+b p_{m, c}+z=0
$$

where by definition,

$$
\begin{gathered}
a=\eta \ln 2 \sum_{m}^{\prime=1} M \sum_{n=1}^{N} \varsigma_{m}{ }^{\prime}\left(1-\rho_{m}{ }^{\prime}\right) s_{m, c} \tilde{\alpha}_{c}^{n}\left|\tilde{h}_{m^{\prime}}^{n}\right|^{2}-\lambda s_{m, c} \ln 2, \\
b=L \mu_{m}-\sum_{\tilde{\alpha}_{c}^{n}=0, n=1, \cdots, N-N_{e}^{m}} u_{m, c}^{n} \\
z=-\sum_{\tilde{\alpha}_{c}^{n} \neq 0, n=1, \cdots, N-N_{e}^{m}} u_{m, c}^{n} \frac{\sigma^{2}}{\tilde{\alpha}_{c}^{n} s_{m, c}\left|\tilde{h}_{e}^{n}\right|^{2}} .
\end{gathered}
$$

Consequently, from (29), we can readily derive that the optimal $p_{m, c}^{*}$ is

$$
p_{m, c}^{*}=\frac{-b+\sqrt{b^{2}-4 a z}}{2 a}, \quad m=1, \cdots, M, c=1, \cdots, C .
$$

Having obtained $p_{m, c}^{*}$ as shown above, the dual problem of (27) can be solved by adopting the subgradient method, owing to the fact that the dual function in (27) is always convex. With this approach, the dual variables are updated using the following formulas:

$$
\mu_{m}(t+1)=\left[\mu_{m}(t)+\kappa_{m}(t)\left(R_{m}^{\mathrm{req}}-R_{m}\right)\right]^{+}, \quad m=1, \cdots, M,
$$

$$
\varsigma_{m}(t+1)=\left[\varsigma_{m}(t)+\theta_{m}(t)\left(R_{m}^{\mathrm{req}}-R_{m}\right)\right]^{+}, \quad m=1, \cdots, M,
$$

$$
\lambda(t+1)=\left[\lambda(t)+\kappa_{0}(t)\left(P_{s}-P_{s}\right)\right]^{+},
$$

where $\kappa_{m}(t)(m=0,1, \cdots, M)$ and $\theta_{m}(t)(m=1,2, \cdots, M)$ are diminishing and small positive step sizes.

To summarize, the iterative algorithm described above for solving the optimization problem of (13a), (13b), (13c), (13d), and (13e) can be stated as Algorithm 1, where $T$ denotes the maximum number of predefined iterations.

\section{Performance Results and Discussion}

Let us now provide some results to demonstrate the SSR performance of the SCMA system, when employing the optimal RA and PS considered in the previous section. In our studies, we assume that the channels experience both path loss and fast fading, with the channel gains expressed as $h_{i}=d_{i}^{-\beta / 2} g_{i}, i \in\{1, \cdots, M, e\}$, where $d_{i}$ and $\beta$ denote the distance between BS and a mobile user (or the eavesdropper) and the path-loss exponent, respectively, while $g_{i}$ obeys the Rayleigh fading. Correspondingly, the parameters for SWIPT are set to $\beta=3, d_{i}=50 \mathrm{~m}$, and $g_{i}$ $\sim C N(0,1)$. Furthermore, we set $\eta=0.8$ and $\sigma^{2}=-70$ $\mathrm{dBm}$. Note that, in our simulations, provided that there exists a user, whose rate or harvested energy is unable to meet the minimum requirements, the SSR of the system is assumed to be zero.

For SCMA, the common parameters are set to $M=6, C$ $=6, N=4, L=2$, and $\alpha_{c}^{n}=0.45$ if $\alpha_{c}^{n} \neq 0$. Moreover, the mapping of $\mathbf{q}$ between codebooks and subcarriers is given by [37]

$$
\mathbf{q}=\left[\begin{array}{llllll}
1 & 1 & 1 & 0 & 0 & 0 \\
1 & 0 & 0 & 1 & 1 & 0 \\
0 & 1 & 0 & 1 & 0 & 1 \\
0 & 0 & 1 & 0 & 1 & 1
\end{array}\right] .
$$

Figure 3 depicts the average SSR versus the BS transmit power $P_{s}$ performance of the SCMA systems. According to [32], we set the constraints $E_{m}^{\mathrm{req}}=50 \mu \mathrm{W}$ and $R_{m}^{\mathrm{req}}=40 \mathrm{bit} / \mathrm{s} /$ Hz. Explicitly, when $30<P_{s}<50 \mathrm{dBm}$, the average SSR achievable by the SCMA systems employing our proposed PA and PS can be much higher than that by the SCMA systems with uniform PA and random codebook assignment. Specifically, at $P_{s}=36 \mathrm{dBm}$, the SSR gain achieved by the optimized SCMA is $1132 \%$, relative to that achieved by the fixed SCMA. However, if the BS transmit power is very high, such as $P_{s}>50 \mathrm{dBm}$, uniform PA and random codebook assignment become efficient, which can be explained as follows. When $P_{s}$ is sufficiently high, uniform PA and random codebook assignment are capable of making the SCMA systems achieve full secrecy capacity. This can be seen from Equation (12), which converges to a fixed value as $P_{s}$ increases. 


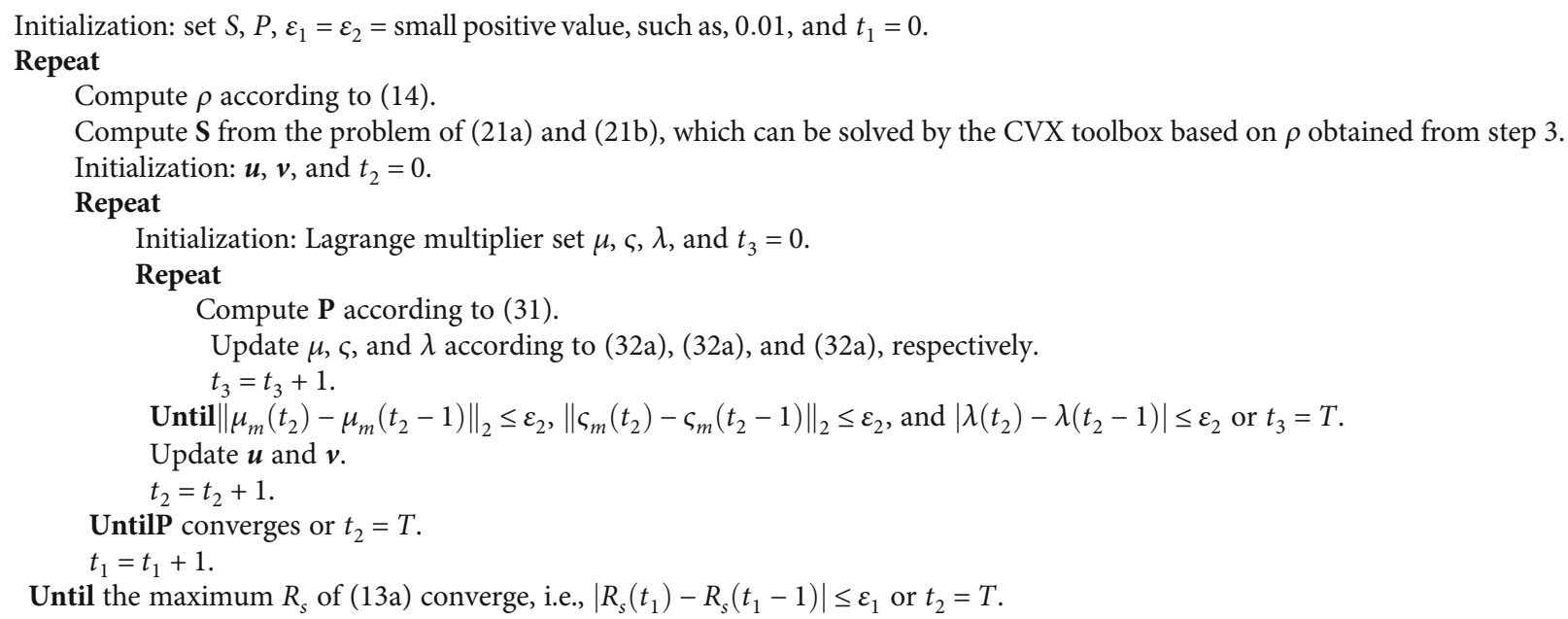

Algorithm 1: Iterative RA algorithm for SCMA systems.

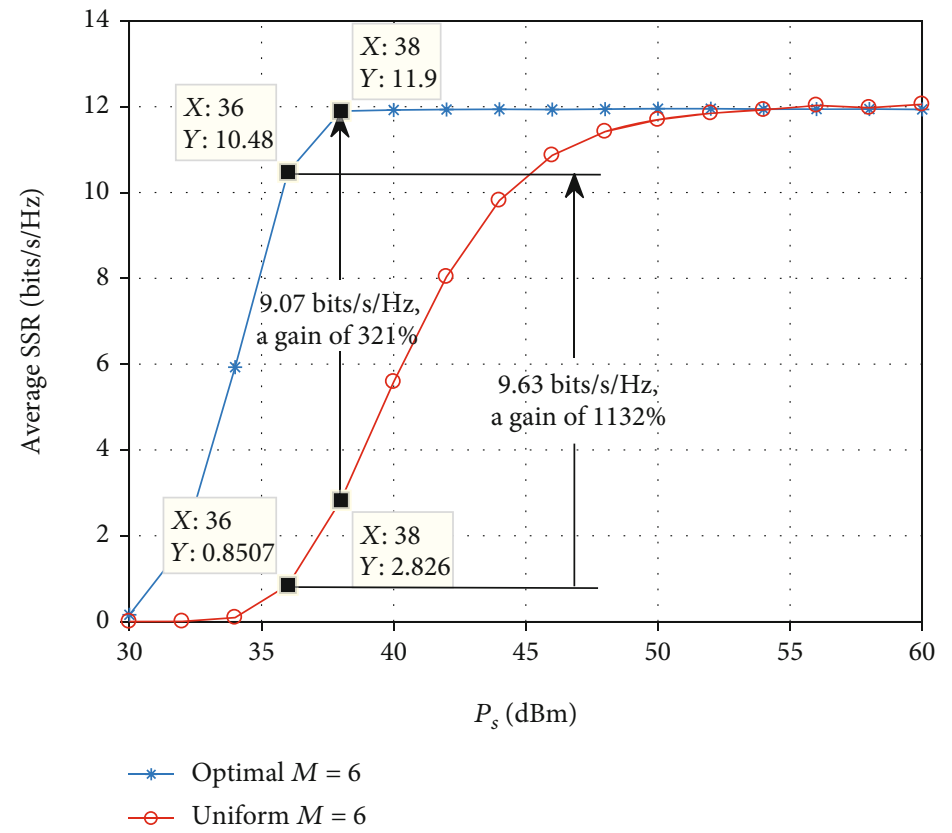

FIGURE 3: Average SSR versus $P_{s}$ performance of SCMA systems with the constraints of $E_{m}^{\text {req }}=50 \mu \mathrm{W}$ and $R_{m}^{\text {req }}=40 \mathrm{bit} / \mathrm{s} / \mathrm{Hz}$.

Figure 4 shows the average SSR of SCMA systems versus the minimum rate, $R_{m}^{\text {req }}$, required by each of the $M$ users. The results show that, as the minimum required rate, $R_{m}^{\text {req, }}$ increases, the achievable SSR of the SCMA systems operated in both the scenarios considered decreases. The reason for this phenomenon is that, as $R_{m}^{\text {req }}$ increases, the BS transmit power may become insufficient to satisfy the minimum rate and energy requirements of the users. Furthermore, as seen in Figure 4, if $R_{m}^{\text {req }}$ is too large, the SSR of the SCMA systems with either of the PA and codebook assignment strategies converges to zero. Otherwise, the SSR of the SCMA systems employing our optimized PA and codebook assignment can be significantly higher than that of the SCMA systems with a uniform PA and random codebook assignment.
Finally, Figure 5 addresses the impact of the minimum harvested energy per user, $E_{m}^{\text {req }}$, on the average SSR achievable by the SCMA systems, when our proposed PA and codebook assignment and the uniform PA and random codebook assignment are, respectively, employed. As the results show, when the constraints of $R_{m}^{\text {req }}=40$ bits $/ \mathrm{s} / \mathrm{Hz}$ and $E_{m}^{\mathrm{req}}<5 \mathrm{dBm}$ are considered, the SSR achieved by the SCMA systems employing our optimized strategy is much higher than that of the SCMA systems with uniform PA and random codebook assignment. However, if $E_{m}^{\text {req }}$ is too high, the SSR of both systems approaches zero. Again, this is because, when the requirement for energy harvesting is too heavy, BS is incapable of meeting all the requirements of all the $M$ users. 


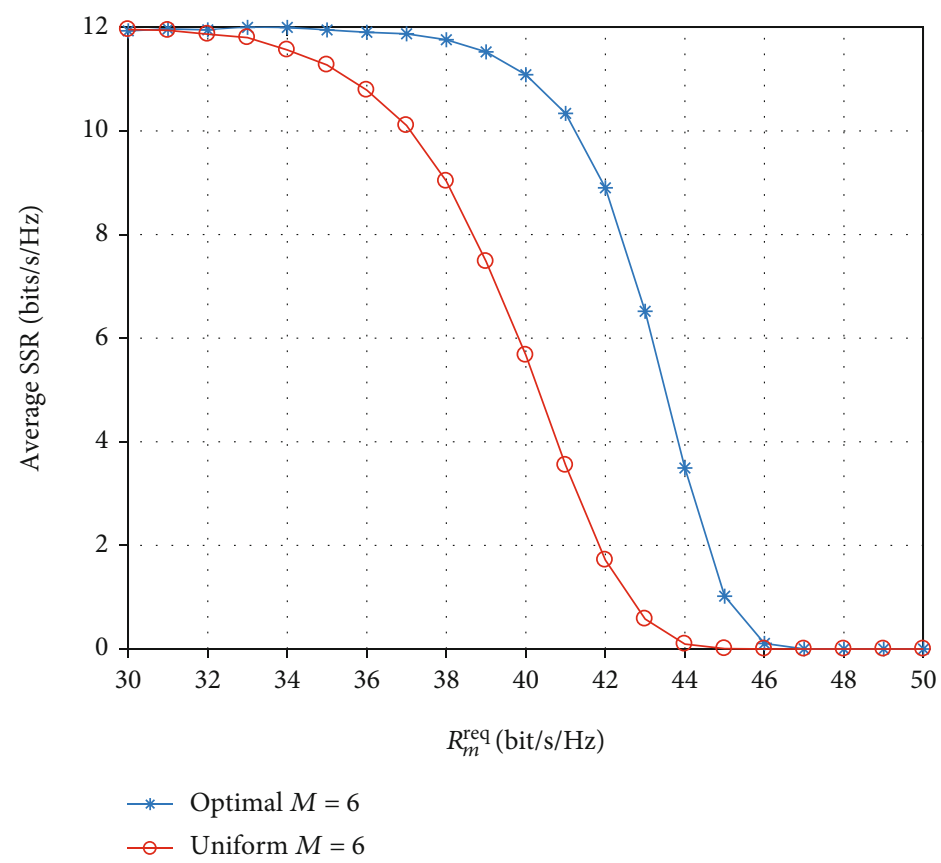

FIGURE 4: Average SSR versus $P_{s}$ performance of SCMA systems with the constraints of $E_{m}^{\text {req }}=50 \mu \mathrm{W}$ and $R_{m}^{\text {req }}=40 \mathrm{bit} / \mathrm{s} / \mathrm{Hz}$.

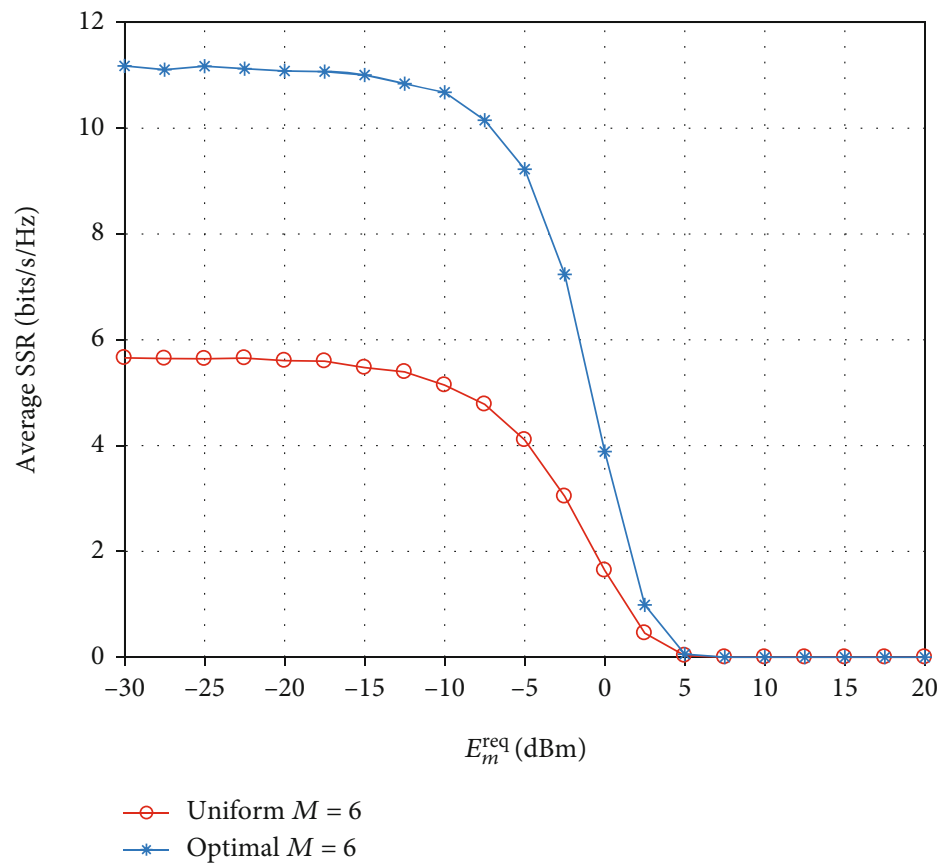

FIGURE 5: Average SSR versus $E_{m}^{\text {req }}$ performance of SCMA systems, when $R_{m}^{\text {req }}=40 \mathrm{bit} / \mathrm{s} / \mathrm{Hz}$ and $P_{s}=40 \mathrm{dBm}$.

\section{Conclusion}

We have designed an optimization framework of SWIPT with SCMA to satisfy the security requirement of 6G AANs. Specifically, we analyzed the PLS of SWIPT in the downlink SCMA system, where RA and PS are employed to coordinate the energy and information delivered to each of the users. In our studies, the PLS and SWIPT have been jointly considered, in order to demonstrate the trade-off between the rate and the harvested energy required by each of the users. Furthermore, the effect of these requirements on the SSR performance has been investigated. From our studies and performance results, the following observations can be obtained. When a SCMA system is operated in association with our proposed PS, codebook assignment, and PA, its achievable SSR performance can be much better than that of the corresponding SCMA system operated with uniform $\mathrm{PA}$ and a random codebook assignment. Moreover, the 
minimum rates or minimum harvested energy demanded by individual users yield a strong impact on their achievable SSR. If the minimum rates or minimum harvested energy required by individual users is too high, there is possibly no SSR achievable. Thus, in practical system design for $6 \mathrm{G}$ ANNs, these requirements must be appropriately set in order for a NOMA system to attain the highest possible SSR.

\section{Data Availability}

The data used to support the study are available within the article.

\section{Conflicts of Interest}

The authors declare that there is no conflict of interest regarding the publication of this article.

\section{Acknowledgments}

This work was supported in part by the National Natural Science Foundation of China (NSFC) under Grant 62001387, in part by the Shanghai Academy of Spaceflight Technology (SAST) under Grant SAST2020-124, and in part by the Fundamental Research Funds for the Central Universities.

\section{References}

[1] P. Yang, Y. Xiao, M. Xiao, and S. Li, "6G wireless communications: vision and potential techniques," IEEE Network, vol. 33, no. 4, pp. 70-75, 2019.

[2] Z. Zhang, Y. Xiao, Z. Ma et al., "6G wireless networks: vision, requirements, architecture, and key technologies," IEEE Vehicular Technology Magazine, vol. 14, no. 3, pp. 28-41, 2019.

[3] N.-N. Dao, Q.-V. Pham, N. H. Tu et al., "Survey on aerial radio access networks: toward a comprehensive $6 \mathrm{G}$ access infrastructure," IEEE Communications Surveys \& Tutorials, 2021.

[4] L. Li, M. Wang, K. Xue et al., "Delay optimization in multiUAV edge caching networks: a robust mean field game," IEEE Transactions on Vehicular Technology, vol. 70, no. 1, pp. 808819, 2021.

[5] L. Li, H. Ren, Q. Cheng et al., "Millimeter-wave networking in the sky: a machine learning and mean field game approach for joint beamforming and beam-steering," IEEE Transactions on Wireless Communications, vol. 19, no. 10, pp. 6393-6408, 2020.

[6] B. Yang, X. Cao, Z. Han, and L. Qian, "A machine learning enabled MAC framework for heterogeneous internet-ofthings networks," IEEE Transactions on Wireless Communications, vol. 18, no. 7, pp. 3697-3712, 2019.

[7] X. Cao, Z. Song, B. Yang, L. Qian, and Z. Han, "Full-duplex MAC in LAA/ Wi-Fi coexistence networks: design, modeling, and analysis," IEEE Transactions on Wireless Communications, vol. 19, no. 8, pp. 5531-5546, 2020.

[8] P. Wang, J. Xiao, and L. Ping, "Correction to "comparison of orthogonal and non-orthogonal approaches to future wireless cellular systems"," IEEE Vehicular Technology Magazine, vol. 1, no. 4, pp. 42-42, 2006.
[9] H. Zhang, J. Zhang, and K. Long, "Energy efficiency optimization for NOMA UAV network with imperfect CSI," IEEE Journal on Selected Areas in Communications, vol. 38, no. 12, pp. 2798-2809, 2020.

[10] Y. Saito, Y. Kishiyama, A. Benjebbour, T. Nakamura, A. Li, and K. Higuchi, "Non-orthogonal multiple access (NOMA) for cellular future radio access," in 2013 IEEE 77th Vehicular Technology Conference (VTC Spring), pp. 1-5, Dresden, Germany, 2013.

[11] Z. Ding, Z. Yang, P. Fan, and H. V. Poor, "On the performance of non-orthogonal multiple access in $5 \mathrm{G}$ systems with randomly deployed users," IEEE Signal Processing Letters, vol. 21, no. 12, pp. 1501-1505, 2014.

[12] Y. Chen, A. Bayesteh, Y. Wu et al., "SCMA: a promising nonorthogonal multiple access technology for 5G networks," in 2016 IEEE 84th Vehicular Technology Conference (VTC-Fall), pp. 1-6, Montreal, QC, Canada, 2016.

[13] L. R. Varshney, "Transporting information and energy simultaneously," in 2008 IEEE International Symposium on Information Theory, pp. 1612-1616, Toronto, ON, Canada, 2008.

[14] Y. Liu, Z. Ding, M. Eikashlan, and H. V. Poor, "Cooperative non-orthogonal multiple access in $5 \mathrm{G}$ systems with SWIPT," in 2015 23rd European Signal Processing Conference (EUSIPCO), pp. 1999-2003, Nice, France, 2015.

[15] X. Lu, P. Wang, D. Niyato, D. I. Kim, and Z. Han, "Wireless networks with RF energy harvesting: a contemporary survey," IEEE Communications Surveys \& Tutorials, vol. 17, no. 2, pp. 757-789, 2015.

[16] L. Li, H. Ma, H. Ren et al., "Learning-aided resource allocation for pattern division multiple access-based SWIPT systems," IEEE Wireless Communications Letters, vol. 10, no. 1, pp. 131-135, 2021.

[17] H. Zhang, M. Feng, K. Long, G. K. Karagiannidis, V. C. M. Leung, and H. V. Poor, "Energy efficient resource management in SWIPT enabled heterogeneous networks with NOMA," IEEE Transactions on Wireless Communications, vol. 19, no. 2, pp. 835-845, 2020.

[18] R. Zhang and C. K. Ho, "MIMO broadcasting for simultaneous wireless information and power transfer," in 2011 IEEE Global Telecommunications Conference - GLOBECOM 2011, pp. 1-5, Houston, TX, USA, 2011.

[19] P. D. Diamantoulakis, K. N. Pappi, Z. Ding, and G. K. Karagiannidis, "Optimal design of non-orthogonal multiple access with wireless power transfer," in 2016 IEEE International Conference on Communications (ICC),, pp. 1-6, Kuala Lumpur, Malaysia, 2016.

[20] Y. Liu, Z. Ding, M. Elkashlan, and H. V. Poor, "Cooperative non-orthogonal multiple access with simultaneous wireless information and power transfer," IEEE Journal on Selected Areas in Communications, vol. 34, no. 4, pp. 938-953, 2016.

[21] D. Zhai, M. Sheng, X. Wang, Y. Li, J. Song, and J. Li, "Rate and energy maximization in SCMA networks with wireless information and power transfer," IEEE Communications Letters, vol. 20, no. 2, pp. 360-363, 2016.

[22] Z. Li, W. Chen, F. Wei, F. Wang, X. Xu, and Y. Chen, "Joint codebook assignment and power allocation for SCMA based on capacity with Gaussian input," in 2016 IEEE/CIC International Conference on Communications in China (ICCC), pp. 1-6, Chengdu, China, 2016. 
[23] L. Lin, L. Li, and S. Xin, "Optimization of resource allocation in relay assisted multi-user SCMA uplink network,” in 2017 International Conference on Computing, Networking and Communications (ICNC), pp. 282-286, Silicon Valley, CA, USA, 2017.

[24] J. Cui, P. Fan, X. Lei, Z. Ma, and Z. Ding, "Downlink power allocation in SCMA with finite-alphabet constraints," in 2017 IEEE 85th Vehicular Technology Conference (VTC Spring), pp. 1-5, Sydney, NSW, Australia, 2017.

[25] A. D. Wyner, "The wire-tap channel," The Bell System Technical Journal, vol. 54, no. 8, pp. 1355-1387, 1975.

[26] Y. Liu, H. Chen, and L. Wang, "Physical layer security for next generation wireless networks: theories, technologies, and challenges," IEEE Communications Surveys \& Tutorials, vol. 19, no. 1, pp. 347-376, 2017.

[27] Z. Qin, Y. Liu, Z. Ding, Y. Gao, and M. Elkashlan, "Physical layer security for $5 \mathrm{G}$ non-orthogonal multiple access in large-scale networks," in 2016 IEEE International Conference on Communications (ICC), pp. 1-6, Kuala Lumpur, Malaysia, 2016.

[28] Y. Liu, Z. Qin, M. Elkashlan, Y. Gao, and L. HanzoEnhancing the physical layer security of non-orthogonal multiple access in large-scale networks," IEEE Transactions on Wireless Communications, vol. 16, no. 3, pp. 1656-1672, 2017.

[29] O. Abbasi and A. Ebrahimi, "Secrecy analysis of a NOMA system with full duplex and half duplex relay," in 2017 Iran Workshop on Communication and Information Theory (IWCIT), pp. 1-6, Tehran, Iran, 2017.

[30] Y. Zhang, H. Wang, Q. Yang, and Z. Ding, "Secrecy sum rate maximization in non-orthogonal multiple access," IEEE Communications Letters, vol. 20, no. 5, pp. 930-933, 2016.

[31] H. Wu, X. Tao, Z. Han, N. Li, and J. Xu, "Secure transmission in MISOME wiretap channel with multiple assisting jammers: maximum secrecy rate and optimal power allocation," IEEE Transactions on Communications, vol. 65, no. 2, pp. 775-789, 2017.

[32] G. He, L. Li, X. Li, W. Chen, L.-L. Yang, and Z. Han, "Secrecy sum rate maximization in NOMA systems with wireless information and power transfer," in 2017 9th International Conference on Wireless Communications and Signal Processing (WCSP), pp. 1-6, Nanjing, China, 2017.

[33] M. Moltafet, N. M. Yamchi, M. R. Javan, and P. Azmi, "Comparison study between PD-NOMA and SCMA," IEEE Transactions on Vehicular Technology, vol. 67, no. 2, pp. 18301834, 2018.

[34] M. Chiang, C. W. Tan, D. P. Palomar, D. O'neill, and D. Julian, "Power control by geometric programming," IEEE Transactions on Wireless Communications, vol. 6, no. 7, pp. 26402651, 2007.

[35] J. Papandriopoulos and J. S. Evans, "SCALE: a low-complexity distributed protocol for spectrum balancing in multiuser DSL networks," IEEE Transactions on Information Theory, vol. 55, no. 8, pp. 3711-3724, 2009.

[36] S. Boyd and L. Vandenberghe, Convex Optimization, Cambridge University Press, Cambridge, UK, 2013.
[37] H. Nikopour and H. Baligh, "Sparse code multiple access," in 2013 IEEE 24th Annual International Symposium on Personal, Indoor, and Mobile Radio Communications (PIMRC), pp. 332336, London, UK, 2013.

[38] A. A. Nasir, X. Zhou, S. Durrani, and R. A. Kennedy, "Relaying protocols for wireless energy harvesting and information processing," IEEE Transactions on Wireless Communications, vol. 12, no. 7, pp. 3622-3636, 2013.

[39] R. Hoshyar, F. P. Wathan, and R. Tafazolli, "Novel low-density signature for synchronous CDMA systems over AWGN channel," IEEE Transactions on Signal Processing, vol. 56, no. 4, pp. 1616-1626, 2008. 